\title{
The Effect of Different Communication Mechanisms on the Movement and Structure of Self-Organised Aggregations
}

\author{
R. Eftimie * \\ Department of Mathematics, University of Dundee, Dundee, DD1 4HN, UK
}

\begin{abstract}
The formation, persistence and movement of self-organised biological aggregations are mediated by signals (e.g., visual, acoustic or chemical) that organisms use to communicate with each other. To investigate the effect that communication has on the movement of biological aggregations, we use a class of nonlocal hyperbolic models that incorporate social interactions and different communication mechanisms between group members. We approximate the maximum speed for left-moving and right-moving groups, and show numerically that the travelling pulses exhibited by the nonlocal hyperbolic models actually travel at this maximum speed. Next, we use the formula for the speed of a travelling pulse to calculate the reversal time for the zigzagging behaviour, and show that the communication mechanisms have an effect on these reversal times. Moreover, we show that how animals communicate with each other affects also the density structure of the zigzags. These findings offer a new perspective on the complexity of the biological factors behind the formation and movement of various aggregations.
\end{abstract}

Keywords and phrases: travelling pulses, zigzag pulses, wave speed, nonlocal hyperbolic system, communication

Mathematics Subject Classification: 92D50, 92-08, 35Lxx

\section{Introduction}

Communication among organisms forms the basis of any social interaction. Social organisms (such as bacteria [42], fish [7], or various mammals [34]) communicate with each other (using chemical, tactile, sound or visual signals) to find food and mates, or to avoid predators. Thus, the formation, persistence and movement of biological aggregations is actually the result of the ways organisms emit/receive information to/from conspecifics via different sensory modalities [6,7,11]. A slightly different type of social communication was investigated by Bode et al. [5], who studied the role of social networks on the structure of aggregations. However, communication alone cannot explain the complexity of spatial and spatio-temporal patterns observed in biological aggregations. A second factor, movementfacilitated social interactions (i.e., movement towards or away from conspecifics, or movement to align with conspecifics), has been investigated intensively in the context of pattern formation (see, for ex-

*Corresponding author. E-mail: reftimie@maths.dundee.ac.uk 
ample, $[2,8,12,13,17,22,24,25,27,29,31,37,45,46,52]$, the review in [41] and references therein). Of these mathematical models that investigate the role of social interactions on pattern formation, only a few models are one-dimensional $[4,8,13,15,32,40]$, with the majority being two- or three-dimensional $[1,9,14,22,29,30,35,46,50]$. Generally, these models consider three social interactions: short-range repulsion, intermediate range alignment/orientation and long-range attraction. These interactions were initially introduced in the seminal papers by Aoki [2] and Reynolds [46], and then adopted by many other models $[12,22,25,29,49,53]$. However, there are also models that ignore the alignment/orientation interactions, and focus only on the repulsive and attractive interactions [23,51], and models which focus, for example, only on the alignment interactions (via velocity matching) [13]. The interplay between these two factors, namely communication and social interactions, was shown to explain the formation of a large variety of aggregative patterns, such as the rippling behaviour in Myxobacteria colonies, the zigzagging motion observed in flocks of birds, or the persistent travelling groups (pulses) displayed by swarms of locusts [17]. Communication mechanisms not only affect the formation or not of a certain type of pattern (behaviour) in animal aggregations, but they can also affect the particular structure of that pattern (behaviour). For example, it was recently shown that moving calls emitted by meerkats lead to an increase in the speed of groups [6]. However, these calls had no effect on the travelling direction of the groups [6].

Investigating the conditions necessary for the existence of travelling pulses (or waves), and in particular the speed of such pulses (or waves) is an active research area in ecology and cell biology. Travelling pulses and waves have been studied extensively in the context of chemotactic interactions modelled by parabolic equations (see, for example, $[28,33,38,47,48]$ and references therein). In the absence of population dynamics (growth and death), these travelling patterns were usually caused by singularities in the chemotactic sensitivity, which lead to unrealistic unbounded velocities. This issue was addressed by focussing on hyperbolic models that incorporate chemotactic sensitivity into the turning rates $[20,36,54]$. In these models, the hyperbolic equations for the movement of cells/organisms were usually coupled with parabolic equations for the production/diffusion of an external chemotactic signal. Moreover, these transport equations modelled only local interactions caused by specific communication mechanisms among cells/organisms (e.g., via concentration levels of succinate and aspartate in [54]). Analytical investigations of such transport models showed that they could exhibit travelling wave solutions even for bounded velocities and turning rates [54]. Further, Lui and Wang [36] investigated the existence of travelling pulses and front patterns in both local hyperbolic models (called by authors "microscopic" models, since they incorporate microscopic-level dynamics, such as individual speed and turning rates) and their limiting local parabolic counterparts (called by authors "macroscopic" models, since the scaling used to obtain them, namely spatio-temporal scaling $\psi=\epsilon x, \tau=\epsilon^{2} t$, with $\epsilon \rightarrow 0$, leads to an averaging of the individual-level dynamics). The authors showed that under certain parametric conditions, travelling wave solutions can be preserved during the transition from these "microscopic" to "macroscopic" models.

While travelling aggregations are very common in nature, the movement is usually interrupted by pauses and sharp reorientations, which allow individuals to explore an area more effectively. The resulting zigzagging behaviour, which in $1 \mathrm{D}$ is characterised by directional movement inter-spread with sharp turns in alternating directions, can often be found in zigzagging flocks of birds [43] or even in swarms of locusts [3]). However, this type of movement is not commonly displayed by continuous mathematical models of biological aggregations, and thus not usually investigated analytically. O'Loan and Evans [40] investigated a similar alternating behaviour in a one-dimensional individual-based model for flocking, and showed numerically that the reversal timescale is proportional to the logarithm of the number of particles in the system. A generalisation of the model in [40] (which included only alignment) to a more complex model that incorporated attraction, repulsion and alignment, showed that the continuum meanfield limit version of the model does not exhibit zigzagging behaviours [44]. Dossetti [15] also investigated zigzagging behaviour in a 1D individual-based model, and showed numerically that transitions between travelling pulses and zigzagging pulses can be explained by the presence of noise and the differences in the interaction radius of individuals. 
In this article, we consider a class of nonlocal hyperbolic models for self-organised biological aggregations (i.e., aggregations that form in the absence of a leader or external signals), and investigate the role of communication mechanisms on the speed of travelling pulse solutions and the reversal time for zigzagging groups. These models, which were initially introduced in $[17,18]$, allow for a straightforward incorporation into the turning rates and into the speed [16] of various social interactions (repulsion from nearby neighbours, attraction towards far-away conspecifics, and alignment with conspecifics at intermediate distances), as well as animal communication based on different sensory modalities (e.g., via sound, tactile, visual or chemotactic signals). These communication mechanisms describe the situation in which an individual receives full or partial information about the location and direction of movement of its neighbours. It was previously shown that the structure of some of the patterns exhibited by these nonlocal hyperbolic models is influenced by social interactions (e.g., larger alignment interactions lead to more dense aggregations in case of stationary groups, and less dense aggregations in case of moving groups [19]). Here, we investigate the combined effect of communication mechanisms and social interactions on the structure and speed of travelling pulses and zigzagging pulses. We show that groups always travel at the maximum possible speed, which is the same for all models, independent of the communication mechanisms used. However, the communication mechanisms influence the zigzagging behaviour of biological aggregations, and in particular the average speed between changes in the movement direction.

We start in Section 2 by briefly reviewing the nonlocal hyperbolic models introduced in [17]. In Section 3, we discuss the effect of communication mechanisms on the formation of spatially homogeneous steady states. In Section 4, we take a heuristic approach to investigate conditions for the existence of travelling-pulse solutions, and approximate the maximum travelling speed for groups that use different communication mechanisms. Then, in Section 5 we compare these values with the numerical values for the speed. In Section 6 we focus on zigzagging pulses and calculate the reversal time between zigzags. We conclude in Section 7 with a discussion of the results and their biological implications.

\section{Model Description}

The evolution of a population formed of densities of right-moving $\left(u^{+}\right)$and left-moving $\left(u^{-}\right)$individuals (on a one-dimensional domain) is described by the following equations:

$$
\begin{aligned}
& u_{t}^{+}+\gamma u_{x}^{+}=-\lambda^{+}\left[u^{+}, u^{-}\right] u^{+}+\lambda^{-}\left[u^{+}, u^{-}\right] u^{-}, \\
& u_{t}^{-}-\gamma u_{x}^{-}=\lambda^{+}\left[u^{+}, u^{-}\right] u^{+}-\lambda^{-}\left[u^{+}, u^{-}\right] u^{-} .
\end{aligned}
$$

Here, $\gamma$ is the constant speed and $\lambda^{ \pm}$are the density-dependent turning rates. In general, the turning rates have a random component and a directed component [16-18]:

$$
\begin{aligned}
\lambda^{ \pm}\left[u^{+}, u^{-}\right] & =\lambda_{1}+\lambda_{2} f\left(y^{ \pm}\left[u^{+}, u^{-}\right]\right) \\
& =\left(\lambda_{1}+\lambda_{2} f(0)\right)+\lambda_{2}\left(f\left(y^{ \pm}\left[u^{+}, u^{-}\right]\right)-f(0)\right),
\end{aligned}
$$

with $f$ a dimensionless, positive, increasing and bounded functional of the interactions with neighbours. (A function that satisfies all these restrictions is $f(y)=0.5+0.5 \tanh \left(y-y_{0}\right)[17,18]$.) The terms $\lambda_{1}+\lambda_{2} f(0)$ and $\lambda_{2}\left(f\left(y^{ \pm}\right)-f(0)\right)$ describe the baseline random turning rate and the bias turning rate, respectively. When $f(0) \ll 1$, the baseline turning can be approximated by $\lambda_{1}$, and the bias turning can be approximated by $\lambda_{2} f\left(y^{ \pm}\right)$. Here, we assume that the turning rates can be influenced by three types of social interactions (recall that the speed $\gamma$ is assumed constant here): attraction (towards neighbours further away), repulsion (from nearby neighbours) and alignment (with neighbours positioned at intermediate distances). Assuming that the effect of these interactions is additive (and keeping in mind that repulsion and attraction have opposite effects), we can define the dimensionless functionals $y^{ \pm}$as follows $[17,18]$ :

$$
y^{ \pm}\left[u^{+}, u^{-}\right]=y_{r}^{ \pm}\left[u^{+}, u^{-}\right]-y_{a}^{ \pm}\left[u^{+}, u^{-}\right]+y_{a l}^{ \pm}\left[u^{+}, u^{-}\right] .
$$


The repulsive $\left(y_{r}^{ \pm}\right)$, attractive $\left(y_{a}^{ \pm}\right)$and alignment $\left(y_{a l}^{ \pm}\right)$terms are nonlocal, and depend on how individuals receive/emit information from/to their conspecifics positioned at certain distances that allow for social interactions [17]. As an example, individuals that communicate through visual signals likely receive information only from ahead (unidirectional reception). In contrast, individuals that communicate through visual and sound signals can receive information from ahead and behind (omnidirectional reception). However, the information received depends also on the information emitted. Thus, the emitters could send out information in all directions (omnidirectional emission; e.g., via chemical or omnidirectional sound signals), or could send out information in a certain direction, which may depend on their movement orientation (unidirectional emission; e.g., via tactile or unidirectional sound signals). Figure 1 shows four examples of different reception and emission mechanisms, which are denoted by M2-M5. These examples were previously introduced in [17]. (In $[17,18]$ the authors considered also a toy model M1, which incorporated the assumption that attraction/repulsion was influenced by all individuals, as in M2, while alignment was influenced only by some particular individuals, as in M4. Since model M1 does not bring any new results to this study, we will ignore it here.) Table 1 describes the interaction terms $y_{j}^{ \pm}, j=r, a, a l$, for each of these four models. Kernels $K_{j}(s), j=r, a l, a$, indicate whether the interactions take place inside the repulsive $\left(K_{r}\right)$, attractive $\left(K_{a}\right)$ or alignment $\left(K_{a l}\right)$ ranges. Throughout this study, we will consider kernels of the form $K_{j}(s)=\left(1 / \sqrt{2 \pi m_{j}^{2}}\right) e^{-\left(s-s_{j}^{2}\right) /\left(2 m_{j}^{2}\right)}, j=r, a, a l$, with $m_{j}=s_{j} / 8$. Here, $s_{j}$ represent half the length of the interaction ranges, and $m_{j}$ describe the width of the interaction ranges. Parameters $q_{r}, q_{a}$ and $q_{a l}$, that appear in front of the integrals in Table 1, describe the magnitudes of the repulsive, attractive and alignment interactions, respectively.

We note here that since the reception of signals from neighbours is the most important aspect when it comes to decision making in an individual (e.g., various neighbours could emit visual and auditive signals, but if the receiving individual cannot perceive the visual signals, it will acquire and act only on partial information), throughout the rest of the article we will associate these models with the mechanism by which individuals perceive information from their neighbours.

\begin{tabular}{cl}
\hline Model & Repulsion, Attraction \& Alignment \\
\hline M2 & $y_{r, a}^{ \pm}=q_{r, a} \int_{0}^{\infty} K_{r, a}(s)(u(x \pm s)-u(x \mp s)) d s$ \\
& $y_{a l}^{ \pm}=q_{a l} \int_{0}^{\infty} K_{a l}(s)\left(u^{\mp}(x \pm s)-u^{ \pm}(x \pm s)+u^{\mp}(x \mp s)-u^{ \pm}(x \mp s)\right) d s$ \\
\hline M3 & $y_{r, a}^{ \pm}=q_{r, a} \int_{0}^{\infty} K_{r, a}(s) u(x \pm s) d s$ \\
& $y_{a l}^{ \pm}=q_{a l} \int_{0}^{\infty} K_{a l}(s)\left(u^{\mp}(x \pm s)-u^{ \pm}(x \pm s)\right) d s$ \\
\hline M4 & $y_{r, a}^{ \pm}=q_{r, a} \int_{0}^{\infty} K_{r, a}(s)\left(u^{\mp}(x \pm s)-u^{ \pm}(x \mp s)\right) d s$ \\
& $y_{a l}^{ \pm}=q_{a l} \int_{0}^{\infty} K_{a l}(s)\left(u^{\mp}(x \pm s)-u^{ \pm}(x \mp s)\right) d s$ \\
\hline M5 & $y_{r, a}^{ \pm}=q_{r, a} \int_{0}^{\infty} K_{r, a}(s) u^{\mp}(x \pm s) d s$ \\
& $y_{a l}^{ \pm}=q_{a l} \int_{0}^{\infty} K_{a l}(s) u^{\mp}(x \pm s) d s$ \\
\hline
\end{tabular}

TABLE 1. Repulsive, attractive and alignment interaction terms corresponding to the four reception mechanisms described in Figure 1. Here, $u=u^{+}+u^{-}$is the total density, $q_{r}, q_{a}$ and $q_{a l}$ are the magnitude of the repulsive, attractive and alignment interactions, while $K_{j}(s), j=r, a, a l$, are the interaction kernels. To be consistent with the work in [17], we consider $K_{j}(s)=\frac{1}{2 \pi m_{j}^{2}} \exp (-(s-$ $\left.s_{j}\right)^{2} / 2 m_{j}^{2}$ ), with $j=r, a, a l, m_{j}=s_{j} / 8$, with $s_{j}$ representing half the length of the interaction ranges, and $m_{j}$ giving the width of the interaction ranges.

Figure 2 shows the types of travelling pulses exhibited by models M2-M5. (To obtain these patterns, we discretised model (2.1)-(2.3) using a first-order upwind scheme, with periodic boundary conditions on a finite domain of length $L=10$. The integrals were discretised using Simpson's rule. The initial conditions were small random perturbations of the spatially homogeneous steady states $u^{+}=u^{-}=1$.) An investigation of a restricted parameter region (obtained by fixing all parameters but $q_{a}$ and $q_{r}$ ) showed 


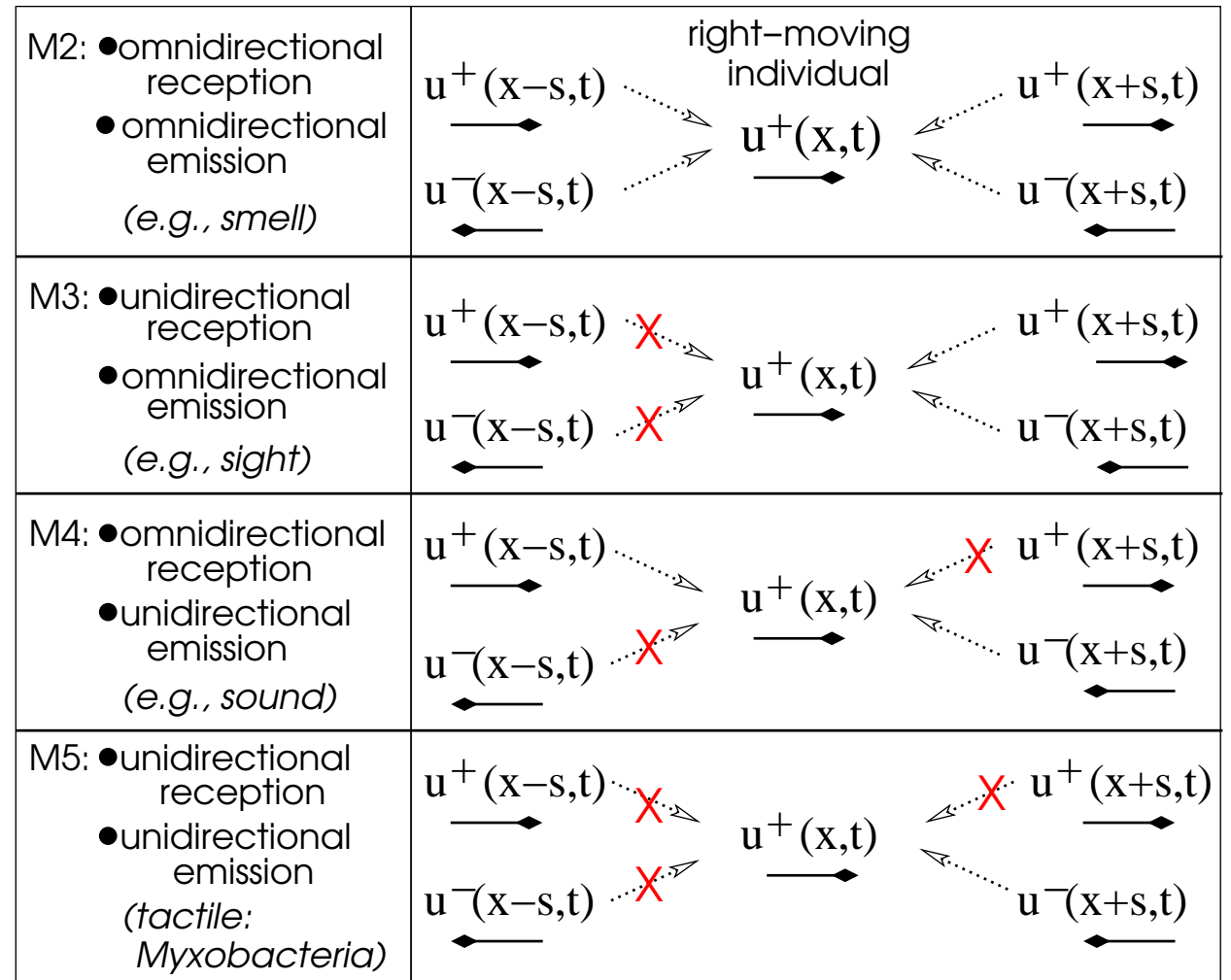

FIGURE 1. Four examples of unidirectional and omnidirectional communication mechanisms, for a right-moving $\left(u^{+}(x, t)\right)$ reference individual that receives information from its neighbours positioned ahead at $x+s$, or behind at $x-s$. Similar mechanisms can be described for a left-moving individual $\left(u^{-}(x, t)\right)$. To avoid confusion, we keep the model notation from [17]: models M2-M5. The continuos arrows describe the direction of movement of individuals (left- or right-moving). The dotted arrows describe the information received from neighbours (where the direction of the arrows indicates the direction in which information travels; e.g., M3: from $u^{ \pm}(x+s, t)$ to $\left.u^{+}(x, t)\right)$. The reference individuals depicted in M2 and M3 can receive information (through visual signals, for example) from neighbours moving towards or away from them. The reference individuals depicted in M4 and M5 can receive information only from neighbours moving towards them. The red (grey on black/white prints) "X" means that no information can be received from those neighbours.

that models M2 and M4 exhibit travelling pulses that are homoclinic orbits corresponding to the state $\left(u^{+}, u^{-}\right) \approx(0,0)$. Models M3 and M5 exhibits travelling pulses that are homoclinic orbits associated with the states $\left(u^{+}, u^{-}\right)=\left(a^{+}, a^{-}\right)$, where $a_{0}^{ \pm}>0$ (see Figures $2(\mathrm{~d}),(\mathrm{h})$.

Throughout most of this article, we will ignore models M3 and M5, and focus only on models M2 and M4 to investigate the speed of the travelling pulses and the reversal times for the zigzagging pulses. Regarding models M3 and M5, there are two observations to make. First, these models do not seem to exhibit zigzagging behaviours [17]. Second, for models M3 and M5, since there is a significant number of individuals spread over the entire domain (see Figure 2(d),(h)), the observed travelling speed of the group (pulse) is a combination of the actual travelling speed and the directional changes of those individuals ahead the group, moving towards the group, who then turn around and look like they are now part of the group. This turning behaviour increases the speed of the group, by artificially moving forward the centre of mass of the group. For these reasons, we will ignore the patterns exhibited by models M3 and M5, and focus only on the patterns exhibited by models M2 and M4. 
(a) model M2

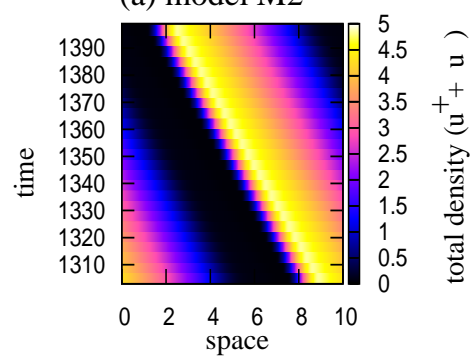

(c) model M3

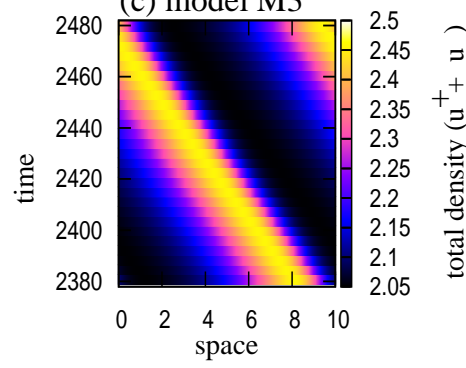

(e) model M4

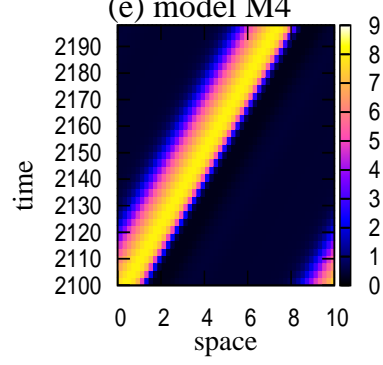

(g) model M5

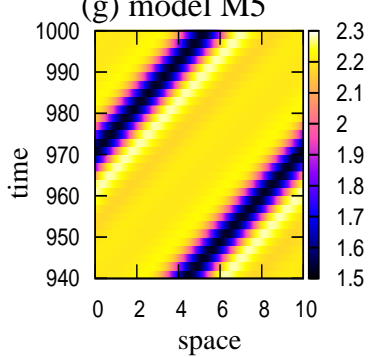

(b) model M2

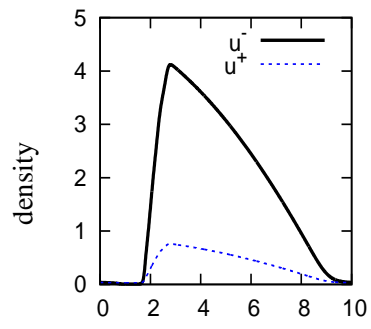

(d) model M3

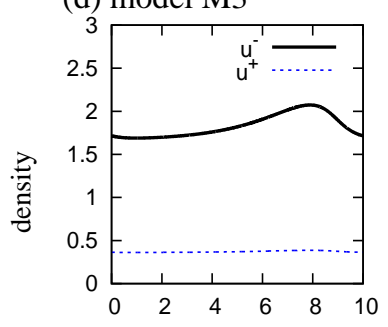

(f) model M4

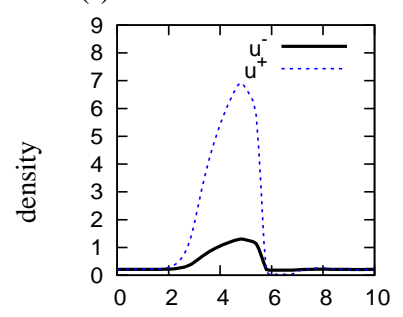

(h) model M5
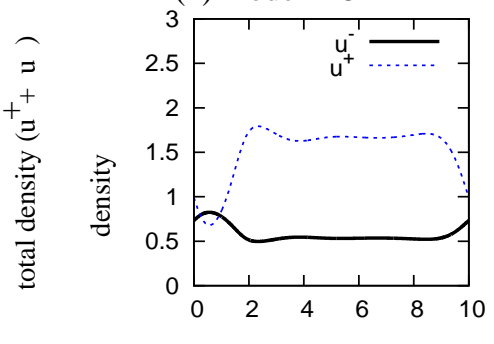

Figure 2. Travelling pulses exhibited by models M2-M5. Panels (a), (c), (e) and (g) show the total density $u=u^{+}+u^{-}$. Panels (b), (d), (f) and (h) show time snapshots of the densities of $u^{+}$and $u^{-}$ separately. The parameters are: (a),(b) $q_{a}=2.1, q_{r}=2$; (c),(d) $q_{a}=2.01, q_{r}=2$; (e),(f) $q_{a}=3.7$, $q_{r}=2 ;(\mathrm{g}),(\mathrm{h}) q_{a}=0.8, q_{r}=0.3$. The rest of parameters are $q_{a l}=2.0, \lambda_{1}=0.2, \lambda_{2}=0.9, \gamma=0.1$, $s_{r}=0.25, s_{a l}=0.5, s_{a}=1.0$.

\section{Spatially homogeneous steady states}

Before investigating the travelling pulses, which are polarised states formed of individuals moving all in one direction, we focus our attention to polarised spatially homogeneous states (i.e., states where there are more individuals facing one direction than the other). 
The spatially homogeneous steady states are solutions of the equation $0=\lambda^{+}\left[u^{+}, u^{-}\right] u^{+}-$ $\lambda^{-}\left[u^{+}, u^{-}\right] u^{-}$. To calculate these states, we substitute $u^{+}=u^{*}, u^{-}=A-u^{*}$ into this equation. (Here $A$ denotes the total population density within a finite domain of length $L: A=\frac{1}{L} \int_{0}^{L}\left(u^{+}(s)+u^{-}(s)\right) d s$. Figure 3 shows these states (for each of the models M2-M5), as functions of the parameter values for the repulsive-attractive $\left(q_{r}-q_{a}\right)$ interactions and for the alignment $\left(q_{a l}\right)$ interactions.

The nonlocal hyperbolic models (2.1) can have one, three or five spatially homogeneous steady states, depending on the communication mechanism used and on the social interactions present (see Figure 3). In particular, all models display the (unpolarised) steady state $u^{ \pm}=u^{*}(=A / 2)$, where half of individuals are moving left and half are moving right. For specific parameter values, the models can exhibit also polarised spatially homogeneous steady states: $\left(u^{+}, u^{-}\right)=\left(A-u^{*}, u^{*}\right)=\left(u^{*}, A-u^{*}\right)$, with $A / 2<u^{*}$ or $u^{*}<A / 2$ (see Figure 3(a)). We can classify these states as "weakly-polarised" states, characterised by $A / 2<u^{*}<1.5$ or $0.5<u^{*}<A / 2$ (as in Figure 3(a), for M5, when $q_{r}-q_{a} \in(1.2,1.5)$ ), and "strongly-polarised" states characterised by $\left(u^{+}, u^{-}\right) \approx(1.7,0.3)$ (as in Figure 3(b), for M2). These "weakly-polarised" and "strongly-polarised" states can coexist in some parameter regions, as shown in Figure 3(a), for M4, when $q_{r}-q_{a} \in(2,3)$.

Note that the social interactions that are mainly attractive (i.e., $q_{r}-q_{a} \ll 0, q_{a l}=0$ ) do not lead to polarised spatially homogeneous steady states (Figure 3(a)). However, polarisation can be obtained by the introduction of alignment interactions $\left(q_{a l}>0\right.$; panel $\left.(\mathrm{b})\right)$.

It was previously shown [17] that in the presence of alignment interactions, all models can display at least three steady states (Figure 3(b,c)). Moreover, the formation of polarised steady states is influenced by the communication mechanisms used. We can see in Figure 3(c) that individuals that are more aware of their neighbours (as in model M2) require less alignment to form polarised states, compared to those individuals that are less aware of their neighbours (as in model M4). Moreover, for small or no alignment, the individuals that are very little aware of their surrounding neighbours (i.e., models M3 and M5), either do not display these polarised states or they display them in very narrow parameter ranges (see Figure $3(\mathrm{a}),(\mathrm{b}))$.

In regard to the stability of these states, note that the state $u^{*}=A / 2=1$ is stable for $q_{r}-q_{a}>0$ (since stronger repulsion impedes the formation of spatio-temporal patterns) and unstable for $q_{r}-q_{a} \ll 0$. Moreover, it was shown in [18] that the state $u^{*}=A / 2$ is always unstable for large $q_{a l}$. On the other hand, the higher-magnitude "strongly-polarised" homogeneous states $\left(u^{*}, A-u^{*}\right) \approx(1.7,0.3)$ are always stable for large $q_{a l}$. Therefore, for large $q_{a l}$, small perturbations of $u^{*}=1$ will likely approach the states $\left(u^{*}, A-u^{*}\right) \approx(1.7,0.3)$. For small to intermediate values of $q_{a l}$, these states could be either stable or unstable (depending on the values of $q_{r, a}$ ). Finally, the "weakly-polarised" states that bifurcate directly form the state $u^{*}=1$ (e.g., the M4-state that bifurcates at $q_{r}-q_{a}=3.2$ in Figure 3(a)) are always unstable [18]. Hence, these states bifurcate subcriticaly from $u^{*}=1$.

Next, we focus on the conditions for the existence of persistent travelling pulses, and investigate the effect of communication mechanisms incorporated into models M2 and M4 on the speed of these pulses.

\section{Conditions for the existence of travel pulse solutions}

We start the investigation into the existence of travelling pulse solutions by considering travelling wave coordinates $z=x-c t$, and defining $U^{ \pm}(z)=u^{ \pm}(x-c t)$. The ODE system satisfied by $U^{ \pm}(z)$,

$$
\begin{aligned}
(\gamma-c) U_{z}^{+} & =-\lambda^{+}\left[U^{+}, U^{-}\right] U^{+}+\lambda^{-}\left[U^{+}, U^{-}\right] U^{-} \\
(-\gamma-c) U_{z}^{-} & =\lambda^{+}\left[U^{+}, U^{-}\right] U^{+}-\lambda^{-}\left[U^{+}, U^{-}\right] U^{-}
\end{aligned}
$$

has the steady state $\left(U^{+}, U^{-}\right)=(0,0)$. Thus, a travelling wave pulse should satisfy $\lim _{z \rightarrow \pm \infty} U^{ \pm}(z)=0$ and $\lim _{z \rightarrow \pm \infty} U_{z}^{ \pm}(z)=0$. Adding and subtracting equations (4.1) leads to

$$
\begin{aligned}
& -c U_{z}+\gamma V_{z}=0, \\
& -c V_{z}+\gamma U_{z}=U \alpha[U, V]-V \beta[U, V],
\end{aligned}
$$


(a)

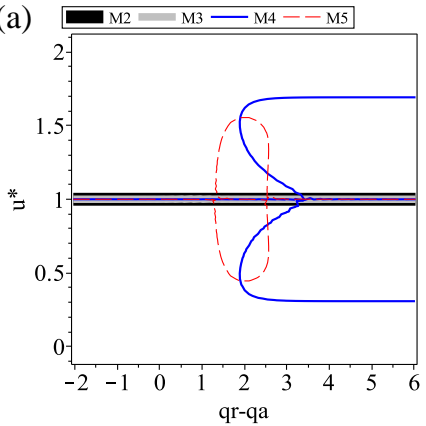

(b)

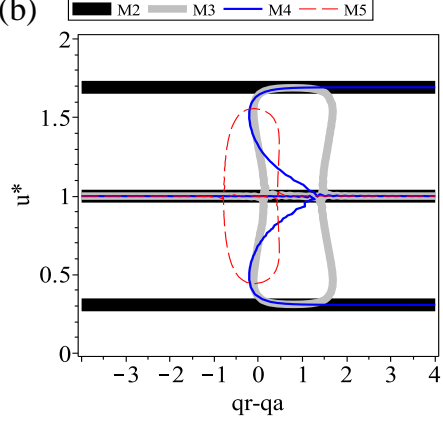

(c)

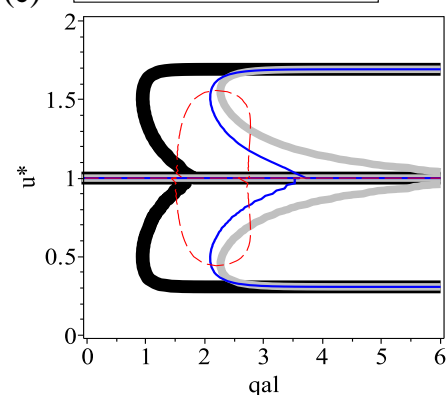

FIGURE 3. Spatially homogeneous steady states $u^{*}$ corresponding to models M2-M5, as functions of the parameters describing the magnitude of the repulsive/attractive interactions $\left(q_{r}-q_{a}\right)$ or the magnitude of alignment interactions $\left(q_{a l}\right)$. (a) $q_{a l}=0$; (b) $q_{a l}=2.1$; (c) $q_{a}=2.2, q_{r}=2.0$. All models have $u^{*}=A / 2$ as a steady state (where $\mathrm{A}$ is the total density of individuals on a domain of length $L$ : $A=\frac{1}{L} \int_{0}^{L}\left(u^{+}(x)+u^{-}(x)\right) d x$. Here and throughout the rest of the paper we choose $\mathrm{A}=2$, and thus $u^{*}=1$.

where $U=U^{+}+U^{-}, V=U^{+}-U^{-}, \alpha[U, V]=\lambda^{-}[U, V]-\lambda^{+}[U, V]$, and $\beta[U, V]=\lambda^{-}[U, V]+\lambda^{+}[U, V]$. From (4.2a) we obtain

$$
V(z)=\frac{c}{\gamma} U(z)
$$

Equation $(4.2 \mathrm{~b})$ can be re-written as

$$
U_{z}=\frac{1}{\gamma^{2}-c^{2}} U\left(\gamma \alpha\left[U, \frac{c}{\gamma} U\right]-c \beta\left[U, \frac{c}{\gamma} U\right]\right) .
$$

A travelling pulse, if it exists, must satisfy

$$
\begin{gathered}
U_{z}(z) \geq 0 \text { for } z \rightarrow-\infty, \\
U_{z}(z) \leq 0 \text { for } z \rightarrow \infty, \\
U(z) \geq 0, \quad \text { for all } z .
\end{gathered}
$$

Note that for non-negative initial data $u_{0}^{ \pm} \in C^{1}$, the solutions $u^{ \pm}$of (2.1) are also non-negative as long as they exist in $C^{1}$. This can be shown by writing equations for $u^{ \pm}$along the characteristic lines, and using the assumption that the turning functions $\lambda^{ \pm}$are positive [19] (see also [26] for an invariance principle for hyperbolic random walk systems). A detailed analysis on the existence of mild, weak and classical solutions for equations (2.1) can be found in [19,21]. Therefore, inequality (4.5c) is satisfied.

To investigate inequalities (4.5a) and (4.5b), we take a heuristic approach. We assume that the pulse is centred at $z=0$ and we approximate the edges of the pulse. In the following, we will focus on a point $z=z_{l}<0$ near the left edge of the pulse (see Figure 4(a)). In the neighbourhood of this point, we have $U\left(z_{l}-s\right) \approx 0$ and $U\left(z_{l}+s\right) \approx B$, with $B$ the constant density of the group. Similarly, if we consider $z=z_{r}>0$ a point near the right edge of the pulse, then $U\left(z_{r}+s\right) \approx 0$ and $U\left(z_{r}-s\right) \approx B$ (see Figure 4(a)). We assume here that the densities near the left and right edges of the pulse have the same value $B$. Thus, we can approximate the edges of the travelling pulse with constant piecewise functions. (Shortly, we will discuss the case where the edges are approximated by exponential functions, as in Figure 4(b).) Using this approach, we can approximate the values of the integral terms $Y_{l, r}^{ \pm}=y^{ \pm}\left[U^{+}\left(z_{l, r}\right), U^{-}\left(z_{l, r}\right)\right]$ (equations (2.3)), which describe the nonlocal interactions for each of the models M2 and M4. These values are given in Table 2.

Using equation (4.4), the approximations for $U(z \pm s)$ and the expressions for $\alpha[U, V]$ and $\beta[U, V]$, we can rewrite inequalities $U_{z}(z)>0$ (near the left edge) and $U_{z}(z)<0$ (near the right edge) as follows: 


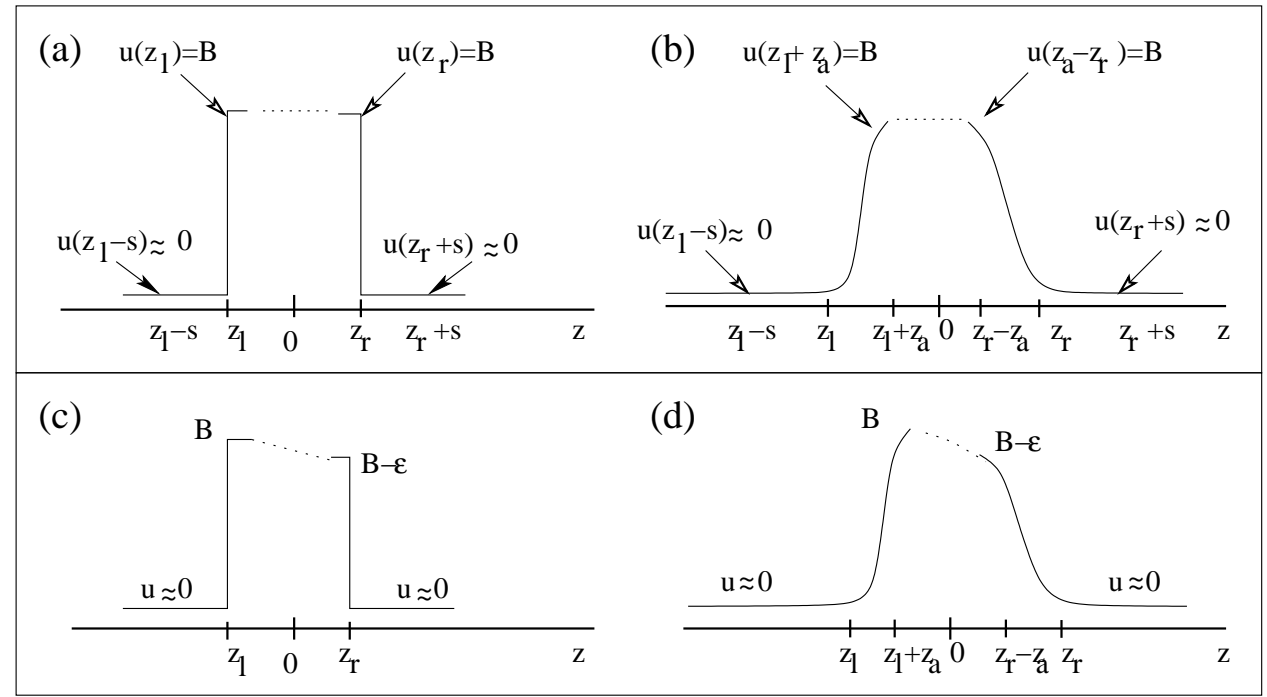

FIGURE 4. Caricature representation of a travelling pulse. (a) Approximation of left/right edges of a pulse with constant piecewise functions with maximum $B$; (b) Approximation of left/right edges of a pulse with exponential functions (equations (4.12)-(4.13)) with maximum $B$. Here, $z_{l, r} \pm z_{a}$ are points at the edge of the attraction zones centred at $z_{l, r}$. There are no social interactions outside these zones; (c) and (d) For more realistic approximations of traveling pulses, we can choose lower densities $(B-\epsilon)$ at the right or left edges of the groups.

\begin{tabular}{cl}
\hline \hline Model & Approximations of nonlocal interactions \\
\hline M2 & $Y_{l}^{-}=-B\left(q_{r}-q_{a}\right)+q_{a l} \frac{c}{\gamma} B$, \\
& $Y_{l}^{+}=B\left(q_{r}-q_{a}\right)-q_{a l} \frac{c}{\gamma} B$, \\
& $Y_{r}^{-}=B\left(q_{r}-q_{a}\right)+q_{a l} \frac{c}{\gamma} B$, \\
& $Y_{r}^{+}=-B\left(q_{r}-q_{a}\right)-q_{a l} \frac{c}{\gamma} B$. \\
\hline M4 & $Y_{l}^{-}=\left(q_{r}-q_{a}+q_{a l}\right)\left(-0.5 B\left(1-\frac{c}{\gamma}\right)\right)$, \\
& $Y_{l}^{+}=\left(q_{r}-q_{a}+q_{a l}\right)\left(0.5 B\left(1-\frac{c}{\gamma}\right)\right)$, \\
& $Y_{r}^{-}=\left(q_{r}-q_{a}+q_{a l}\right)\left(0.5 B\left(1+\frac{c}{\gamma}\right)\right)$, \\
& $Y_{r}^{+}=\left(q_{r}-q_{a}+q_{a l}\right)\left(-0.5 B\left(1+\frac{c}{\gamma}\right)\right)$. \\
\hline \hline
\end{tabular}

TABLE 2. Nonlocal interaction terms for the left $\left(Y_{l}^{ \pm}\right)$and the right $\left(Y_{r}^{ \pm}\right)$edges of a rectangular traveling pulse, for the communication mechanisms M2 and M4 described in Figure 1.

$$
\begin{aligned}
& -2 c \lambda_{1}+(\gamma-c) \lambda_{2} f\left(Y_{l}^{-}\right)-(\gamma+c) \lambda_{2} f\left(Y_{l}^{+}\right) \geq 0, \quad \text { for } \quad z=z_{l}<0 \\
& -2 c \lambda_{1}+(\gamma-c) \lambda_{2} f\left(Y_{r}^{-}\right)-(\gamma+c) \lambda_{2} f\left(Y_{r}^{+}\right) \leq 0, \quad \text { for } \quad z=z_{r}>0
\end{aligned}
$$

with $Y_{l}^{ \pm}$and $Y_{r}^{ \pm}$describing the nonlocal interactions near the left and right edges of the travelling group, respectively (see Table 2). Note that, since $\gamma \geq c$ (individual's speed should be larger than group speed [36]), inequalities (4.6) are not influenced by the sign of $\gamma-c$. Because of the alignment interaction terms, which depend on the speed $c$ (since $\left.U^{ \pm}=0.5(U \pm V)=0.5(U \pm c U / \gamma)\right), Y_{l, r}^{ \pm}$will also depend on the speed $c$. Keeping in mind this aspect, we can rewrite equations (4.6) as follows: 


$$
\begin{gathered}
\frac{\gamma \lambda_{2}\left(f\left(Y_{l}^{-}\right)-f\left(Y_{l}^{+}\right)\right)}{2 \lambda_{1}+\lambda_{2}\left(f\left(Y_{l}^{-}\right)+f\left(Y_{l}^{+}\right)\right)} \geq c, \\
\frac{\gamma \lambda_{2}\left(f\left(Y_{r}^{-}\right)-f\left(Y_{r}^{+}\right)\right)}{2 \lambda_{1}+\lambda_{2}\left(f\left(Y_{r}^{-}\right)+f\left(Y_{r}^{+}\right)\right)} \leq c .
\end{gathered}
$$

Define

$$
c^{*}(c)=\frac{\gamma \lambda_{2}\left(f\left(Y_{l}^{-}\right)-f\left(Y_{l}^{+}\right)\right)}{2 \lambda_{1}+\lambda_{2}\left(f\left(Y_{l}^{-}\right)+f\left(Y_{l}^{+}\right)\right)},
$$

and

$$
c^{* *}(c)=\frac{\gamma \lambda_{2}\left(f\left(Y_{r}^{-}\right)-f\left(Y_{r}^{+}\right)\right)}{2 \lambda_{1}+\lambda_{2}\left(f\left(Y_{r}^{-}\right)+f\left(Y_{r}^{+}\right)\right)} .
$$

Thus, if a travelling pulse exists, its speed $c$ must satisfy the inequality

$$
c^{* *}(c) \leq c \leq c^{*}(c) .
$$

Since for large $Y_{l, r}^{ \pm}$(i.e., large social interactions or large $B$ ) the turning functions can be approximated by $f \approx 1$ or $f \approx 0$, one can easily calculate the maximum and minimum values of $c$ :

$$
c_{\max , \min }= \pm \frac{\gamma \lambda_{2}}{2 \lambda_{1}+\lambda_{2}} .
$$

For $\gamma=0.1, \lambda_{1}=0.2$ and $\lambda_{2}=0.9$ (parameter values that are fixed throughout this article) we obtain $c_{\max , \min } \approx \pm 0.069231$. Therefore, the maximum travelling speed does not depend on the communication mechanism used by individuals in the group.

Figure 5 shows the solutions of equations $c^{* *}(c)=c$ (dotted curves) and $c^{*}(c)=c$ (continuous curves), under the assumptions that (i) groups have well-defined edges and there are no individuals outside the groups, (ii) all groups have the same density $B$, independent of the communication mechanisms used, and (iii) the groups are similar to the one described in Figure 4(a) (i.e., rectangular shape and same density $B$ near the left and right edges of the group). First, we observe that for large or small $q_{a}-q_{r}$, the speed $c$ approaches the maximum and minimum values \pm 0.069231 . Comparing the magnitude of the speed for the M2 and M4 models, we observe that it depends on the maximum group density $B$. For relatively small $B$ (panels (a), (b)) and repulsive aggregations (i.e., $q_{a}-q_{r} \leq 0$ ), the magnitude of the speed is the same for both M2 and M4. In contrast, for attractive aggregations (i.e., $q_{a}-q_{r} \gg 0$ ), the magnitude of the speed for M2 pulses is slightly higher than the magnitude of the speed for M4 pulses. However, for large $B$ (panel (c)) the magnitude of the speed for M2 pulses can be lower than the magnitude of the speed for M4 pulses.

Focusing now on inequality (4.10) and its application to the results in Figure 5, we observe that for model M4, travelling pulses exist when $q_{a}-q_{r} \geq 2$ (since in this case $c^{* *}<c^{*}$ ). However, for model M2, travelling pulses exist when $q_{a} \approx q_{r}$ (for relatively small $B$; Figure $5(\mathrm{a}),(\mathrm{b})$ ), or when $q_{a} \leq q_{r}$ (for large $B$; Figure 5(c)).

\subsection{Pulses with exponentially-decaying edges}

To check whether these results are not an artefact of the approximation of a travelling pulse with a rectangular pulse, we consider also pulses with exponentially-decaying edges (see Figure 4(b)). Again, we assume that these pulses are centred at $z=0$. We choose two points $z_{l}<0$ and $z_{r}>0$ at the left and right edges of the travelling pulse, such that $U\left(z_{l, r}\right) \ll 1$ and we can approximate $U\left(z_{l, r}\right) \approx 0$. Let $z_{a}$ be the length of the attraction range for individuals positioned at $z_{l, r}$. Now, we focus on the point $z_{l}<0$ 

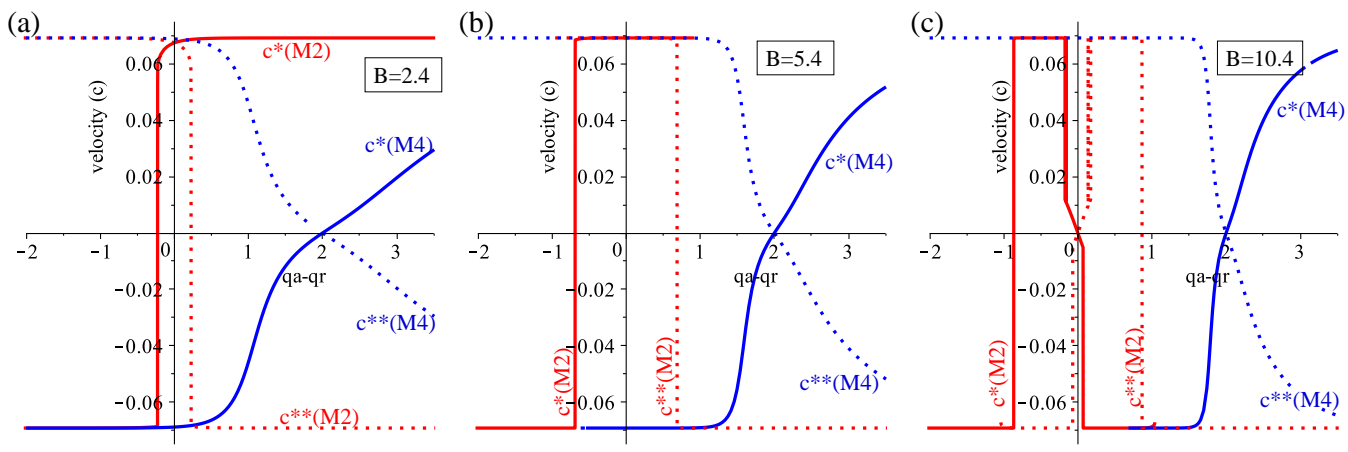

FIGURE 5. Approximations for the maximum and minimum speeds $c$ of travelling pulses of rectangular shapes, as functions of the difference $q_{a}-q_{r}$, for different values of the maximum group density $B$. The continuous curves are solutions of $c=c^{*}(c)$. The dotted curves are solutions of $c=c^{* *}(c)$. (a) $B=2.4$; (b) $B=5.4$; (c) $B=10.4$. The rest of parameters are as follows: $q_{a l}=2.0, \lambda_{1}=0.2, \lambda_{2}=0.9$, $s_{r}=0.25, s_{a l}=0.5, s_{a}=1.0, L=10$.

(Figure $4(\mathrm{~b})$ ), and define $B$ the maximum density at the edge of the attraction zone (i.e., $U\left(z_{l}+z_{a}\right)=B$ ). Then, we can approximate the left edge of the pulse by

$$
U\left(z_{l}+s\right)= \begin{cases}B-B e^{-\frac{B}{b_{l}}(s)^{2}}, & \text { for } 0<s<z_{a} \\ 0, & \text { for } s \leq 0 .\end{cases}
$$

Similarly, if we focus on the point $z_{r}>0$ at the right edge of the pulse, we can define $B$ to be the maximum density at the edge of the attraction zone (i.e., $U\left(z_{r}-z_{a}\right)=B$ ). Thus, we can approximate the right edge of a pulse by

$$
U\left(z_{r}-s\right)= \begin{cases}B-B e^{-\frac{B}{b_{r}}(s)^{2}}, & \text { for } 0<s<z_{a} . \\ 0, & \text { for } s \leq 0\end{cases}
$$

Constants $b_{l}$ and $b_{r}$ give the slope of the left and right edges of the pulses. They are calculated by fitting equations (4.12)-(4.13) to the front edges of the numerical travelling pulses (see Figure 6). For $b_{l, r} \rightarrow 0$, the pulse in Figure 4(b) approaches the rectangular pulse in Figure 4(a).

We can now approximate the speed $c$ by substituting equations (4.12)-(4.13) into the expressions for $y_{r, a l, a}^{ \pm}$in Table 1, calculating the expressions for $Y_{l, r}^{ \pm}$(which now include the convolutions of $U\left(z_{r, l}\right)$ with the interaction kernels $K_{j}(z), j=r, a l, a$; not shown here) and solving numerically the equations for $c^{*}(c)$ and $c^{* *}(c)$. Figure 7 shows these speed approximations for a travelling pulse with exponentially-decaying edges. Compared to the case presented in Figure 5 (where pulses have rectangular edges), here the curves $c^{*}$ and $c^{* *}$ are translated to the left. (This behaviour is the result of having $b_{l}, b_{r}>0$ in equations (4.12)(4.13).) Note that the magnitude of these speed curves is not affected by the approximation chosen. However, the parameter regions where the travelling pulses can exist (i.e., where $c^{* *} \leq c^{*}$ ) are shifted to the left for both M2 and M4 models. We remark that in this case, the M4 model could theoretically display travelling pulses also for negative $q_{a}-q_{r}$.

In the following section, we calculate numerically the speed of travelling pulses, and discuss how appropriate these two approximations are for the real travelling pulses.

\section{Comparison with numerical results}

To test the accuracy of these analytical results, we calculate numerically the speed of travelling pulses. The initial conditions for the numerical simulations are small, random perturbations of the steady states $\left(u^{+}, u^{-}\right)=\left(u^{*}, A-u^{*}\right)$. 

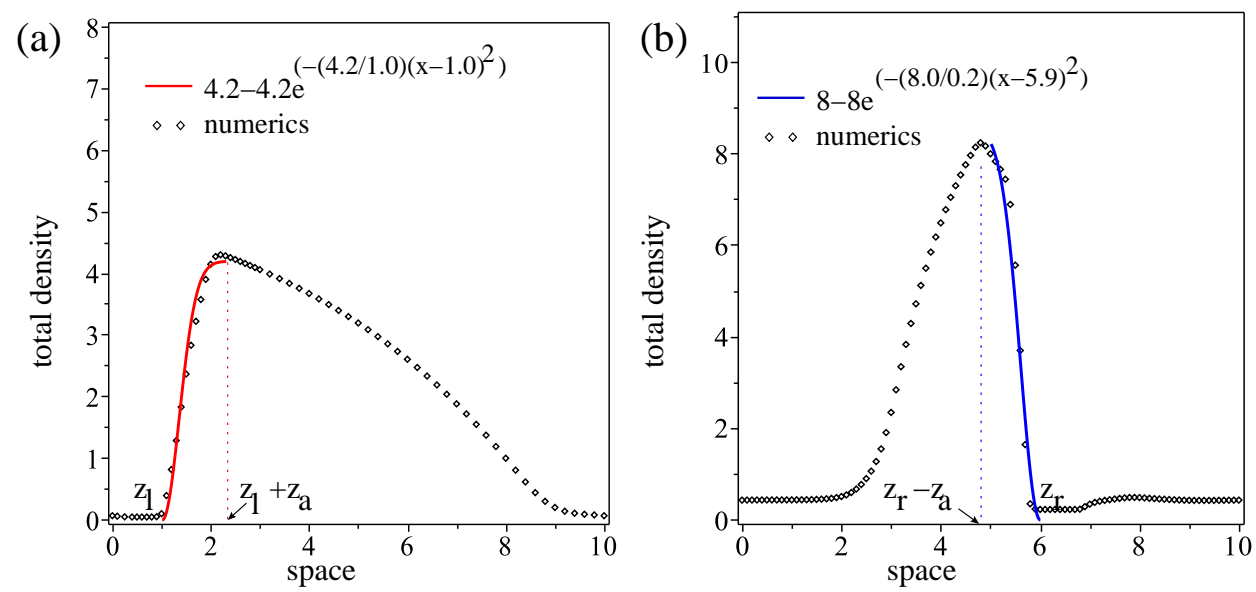

FIGURE 6. Approximations of the left and right edges of the travelling pulses, using equations (4.12)(4.13). (a) Left-moving travelling pulse obtained for model M2, when $q_{a l}=2, q_{a}=2.1, q_{r}=2, B=4$. Here $b_{l}=1.0$; (b) Right-moving travelling pulse obtained for model M4, when $q_{a l}=2, q_{a}=3.7, q_{r}=2$, $B=8$. Here $b_{r}=0.2$.

(a)

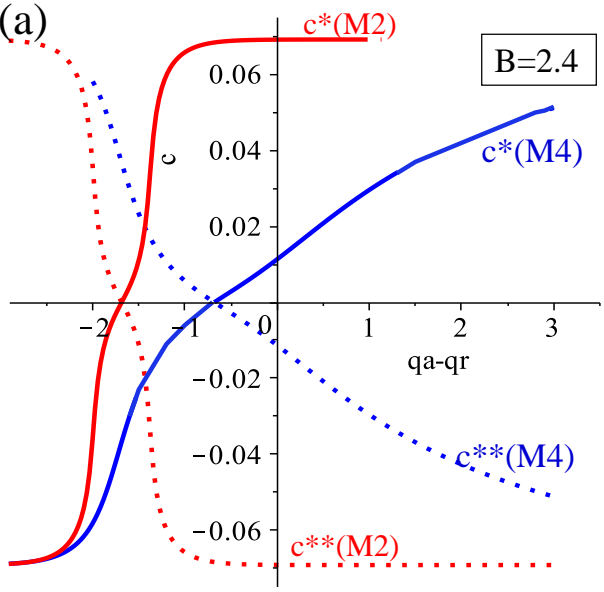

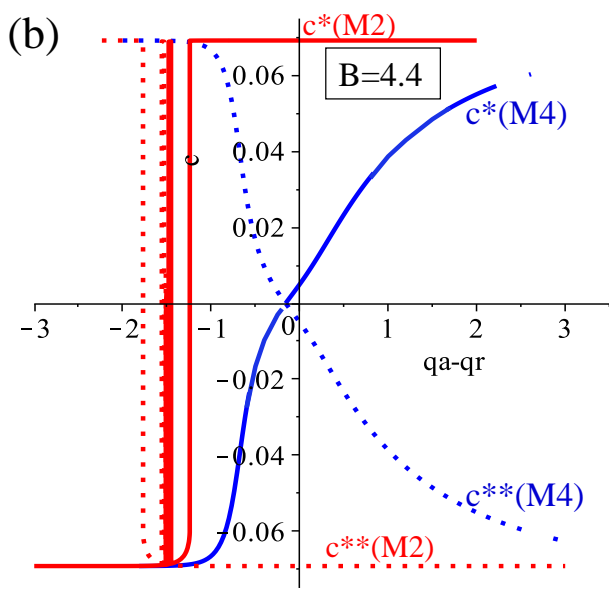

FIGURE 7. Approximations for the maximum and minimum speeds $c$ of travelling pulses with exponentially-decaying edges (given by eq. (4.12)-(4.13)), as functions of the difference $q_{a}-q_{r}$, for different values of $B$. The continuous curves are solutions of $c=c^{*}(c)$. The dotted curves are solutions of $c=c^{* *}(c)$. (a) $B=2.4$; (b) $B=4.4$. The rest of parameters are as follows: $q_{a l}=2.0, \lambda_{1}=0.2$, $\lambda_{2}=0.9, s_{r}=0.25, s_{a l}=0.5, s_{a}=1.0, L=10, b_{l}=1.0, b_{r}=1.0$.

In Figure 8, we graph the approximated values of $c$ (equations (4.8)-(4.9)) versus the numerical values of $c$, for different attractive $\left(q_{a}\right)$ and repulsive $\left(q_{r}\right)$ interactions. Panels (a) and (c) are obtained when we assume rectangular pulses, while panels (b) and (d) are obtained when we assume pulses with exponentially-decaying edges. Since numerical simulations show that the maximum density of the travelling pulses is different for different communication mechanisms, here we calculate the values of $c^{*}$ and $c^{* *}$ by substituting different values of $B$ for each of the models M2 and M4. In particular, to compare with the numerical results, we choose $B$ similar to the values obtained for the numerically simulated travelling pulses: $B \approx 3.5$ for $\mathrm{M} 2$ and $B \approx 8.6$ for M4. Note that choosing slightly different values for $B$ leads to 

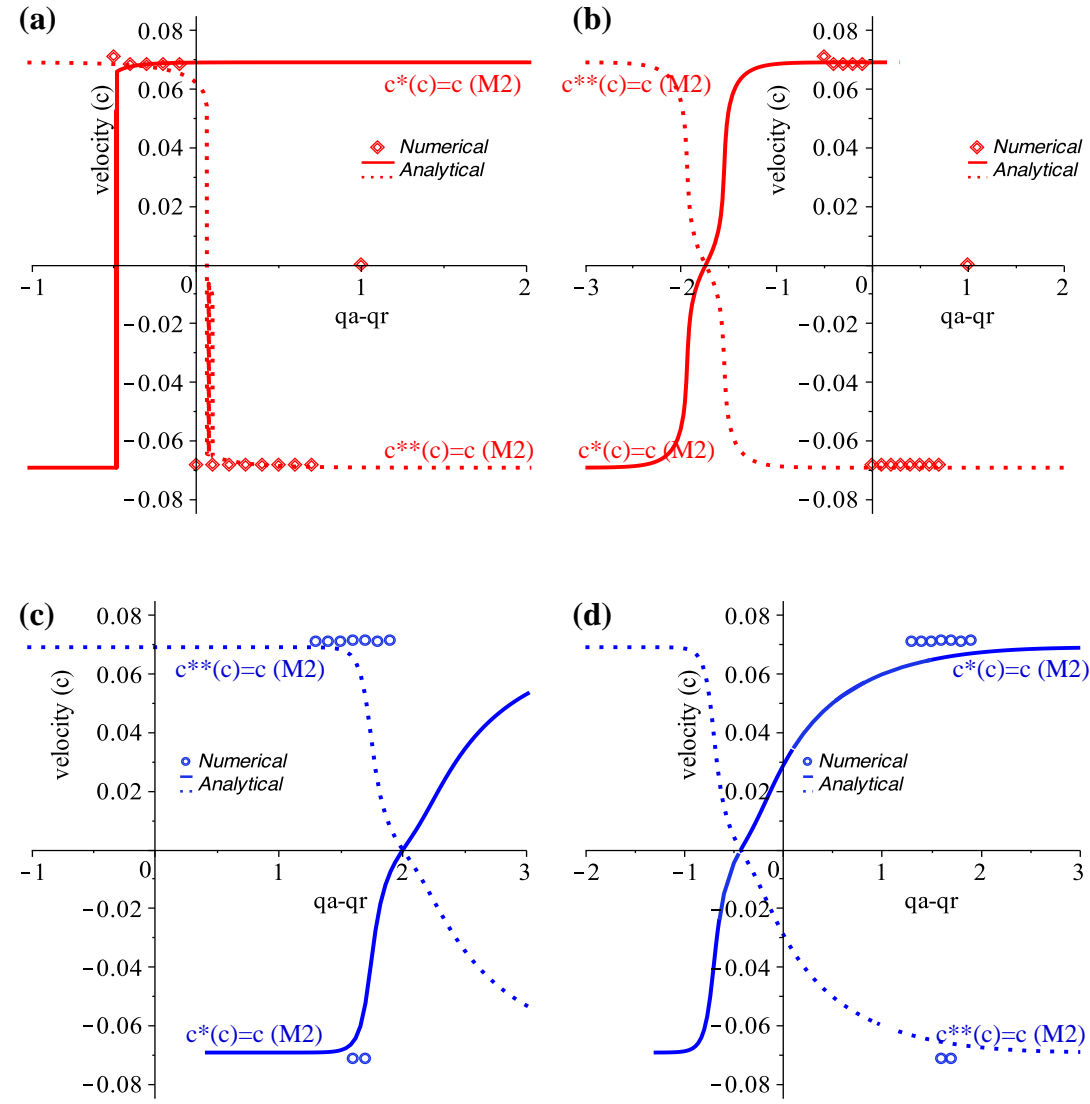

FIGURE 8. Numerical and approximated values for the speed of travelling pulses exhibited by models M2 and M4. In panels (a) and (c) the travelling pulses are approximated by rectangles, while in panels (b) and (d) the pulses have exponentially decaying edges. To calculate the values of $c$ corresponding to $c^{*}(c)=c$ (continuous curves) and $c^{* *}(c)=c$ (dotted curves), we have chosen $B$ such that it is close to the maximum density of the numerically simulated travelling group. Thus, for model M4, $B=8.6$, $b_{l}=b_{r}=0.2$. For model M2, we choose $B=3.5, b_{l}=b_{r}=1.5$. To graph the value of $c$ versus the difference $q_{a}-q_{r}$, we fix $q_{r}$ and vary $q_{a}$. The rest of the parameters are: $q_{a l}=2, q_{r}=2, \lambda_{1}=0.2$, $\lambda_{2}=0.9, s_{r}=0.25, s_{a l}=0.5, s_{a}=1.0, \gamma=1.0, L=10$.

small translations to the left/right for the curves $c^{*}(c)$ and $c^{* *}(c)$. However, these changes do not affect the maximum values of the speed $c$.

Overall, the numerical and analytical values of the travelling speeds $c$ agree relatively well for models M2 and M4, independent of the approximation used. The maximum and minimum values of these curves are consistent for the two approximations, and the M2 and M4 pulses actually travel at the maximum speed $|c| \approx 0.0692$. For model M2, the parameter range in which numerical pulses exist coincides with the parameter range given by $c^{* *} \leq c^{*}(4.10)$, for both pulse approximations (Figure 8(a),(b)). However, for model M4, these parameter ranges coincide only for the approximation with pulses with exponentiallydecaying edges (Figure $8(\mathrm{~d})$ ). When we approximate a travelling pulse with a rectangular-shaped pulse (Figure 8(c)), the analytical results show that this pulse exists for $q_{a}-q_{r}>2$, while the numerical results show that it exists for $q_{a}-q_{r} \leq 2$.

Finally, we remark for model M2 that the numerical speed is negative for $q_{a}-q_{r}>0$ and positive for $q_{a}-q_{r}<0$. The simulations were repeated a few times with the same parameter values (and initial conditions random perturbations of the spatially homogeneous steady states). This raises the question of whether the communication mechanism M2 affects the directionality of the movement. Analytical 
results obtained when the travelling pulse is approximated with a pulse with exponentially decaying edges (Figure $8(\mathrm{~b})$ ) suggest that the curve $c^{*}(c)=c$ does not have real values for $c \gg 0$.

\section{Communication mechanisms and the structure of zigzag pulses}

Another pattern characterised by travelling groups is represented by zigzag pulses, where aggregations change periodically their movement direction. As mentioned previously in [17], both models M2 and M4 can exhibit this type of pattern. Figure 9 shows the types of zigzags obtained when we vary the $q_{a}$ values, while keeping all other parameters fixed. For model M2, one can obtain regular zigzags (panel (a)) as well as a period-4 zigzags (panel (b)), period-2 zigzags (panels (c),(f)) and period-10 zigzags (panel (d)). Note that the formation of these zigzag patterns and the transitions between these different types of zigzags that occur as $q_{a}$ is varied, are the result of symmetry-breaking bifurcations of $\mathbb{Z}_{2}(\kappa, \pi)$ invariant solutions. A detailed investigation of the mechanisms behind these bifurcations will be the subject of a future study. (The role of model symmetries on the bifurcation dynamics of complex patterns exhibited by these nonlocal hyperbolic models has started to be investigated only recently [10].) In regard to the reversal frequency, we remark that for both M2 and M4 models, small $q_{a}$ leads to less frequent changes in the movement direction (panels (b), (c), (d), (f)), compared to the case of large $q_{a}$ (panels (a), (e)). Finally, note that for model M2 the density is higher during the turning process (Figure 9 (c),(d)). In contrast, for model M4, the density reaches its maximum immediately after the group starts reversing (Figure 9(e)). This aspect can be observed more clearly in Figure 10, where we show the surface contour of the zigzags.

In an article that discussed zigzag movement in an individual-based model for flocking, O'Loan and Evans [40] showed that reversal time is directly proportional to the logarithm of the group size. Moreover, the authors suggested that the role of directional changes is to keep the group together: if the group would not reverse its direction, it would become diffuse and cease to exist [40]. Figure 9(e) shows clearly that the back of the group becomes quite diffuse just before the group changes its movement direction, thus supporting the idea put forward in [40].

By direct comparison of the patterns exhibited by models M2 (Figure 9(a)-(d)) and M4 (Figure 9(e)(f)), we also observe that the M4-zigzags are longer than the M2-zigzags. The total group density (which is higher for M2 than for M4) certainly plays a role in this behaviour. If we would assume that the communication mechanisms do not affect the reversal times, since all parameters but $q_{r}$ are the same for both models, the observed patterns would suggest that the difference in the length of the zigzag is the result of the repulsion force, which is higher for M4 $\left(q_{r}=2\right)$ than for M2 $\left(q_{r}=1\right)$. However, a higher repulsion force means that the M4-groups would disperse faster (since the attraction force is the same), which would imply that the zigzags should be shorter, to compensate for the dispersal. This contradiction with the actual length of the zigzags suggests that the communication mechanisms (M2 and M4) play an important role in the reversal times and the type of the zigzags observed numerically.

To calculate the reversal time, we use the formula

$$
T=\frac{d}{c_{a}}
$$

where $d$ is the distance travelled between reversals, and $c_{a}$ is the average travelling speed. Note that between reversals, the groups behave like travelling pulses that move at a constant speed (since the travelled path is rectilinear; see Figure 9). However, because the group slows down to change the movement direction, the average speed $c_{a}$ should be lower than the travelling speed $c \approx 0.069231$ given by equation (4.11).

To calculate the distance $d$, we return to the travelling wave equation (4.4), which we integrate from $z_{0}$ to $z_{1}$, two generic spatial points at which two consecutive reversals occur (see Figure 10):

$$
\int_{z_{0}}^{z_{1}} \frac{U_{z}}{U} d z=\int_{z_{0}}^{z_{1}} \frac{1}{\left(\gamma^{2}-c_{a}^{2}\right)}\left(\gamma \alpha\left[U, \frac{c_{a}}{\gamma} U\right]-c_{a} \beta\left[U, \frac{c_{a}}{\gamma} U\right]\right) .
$$



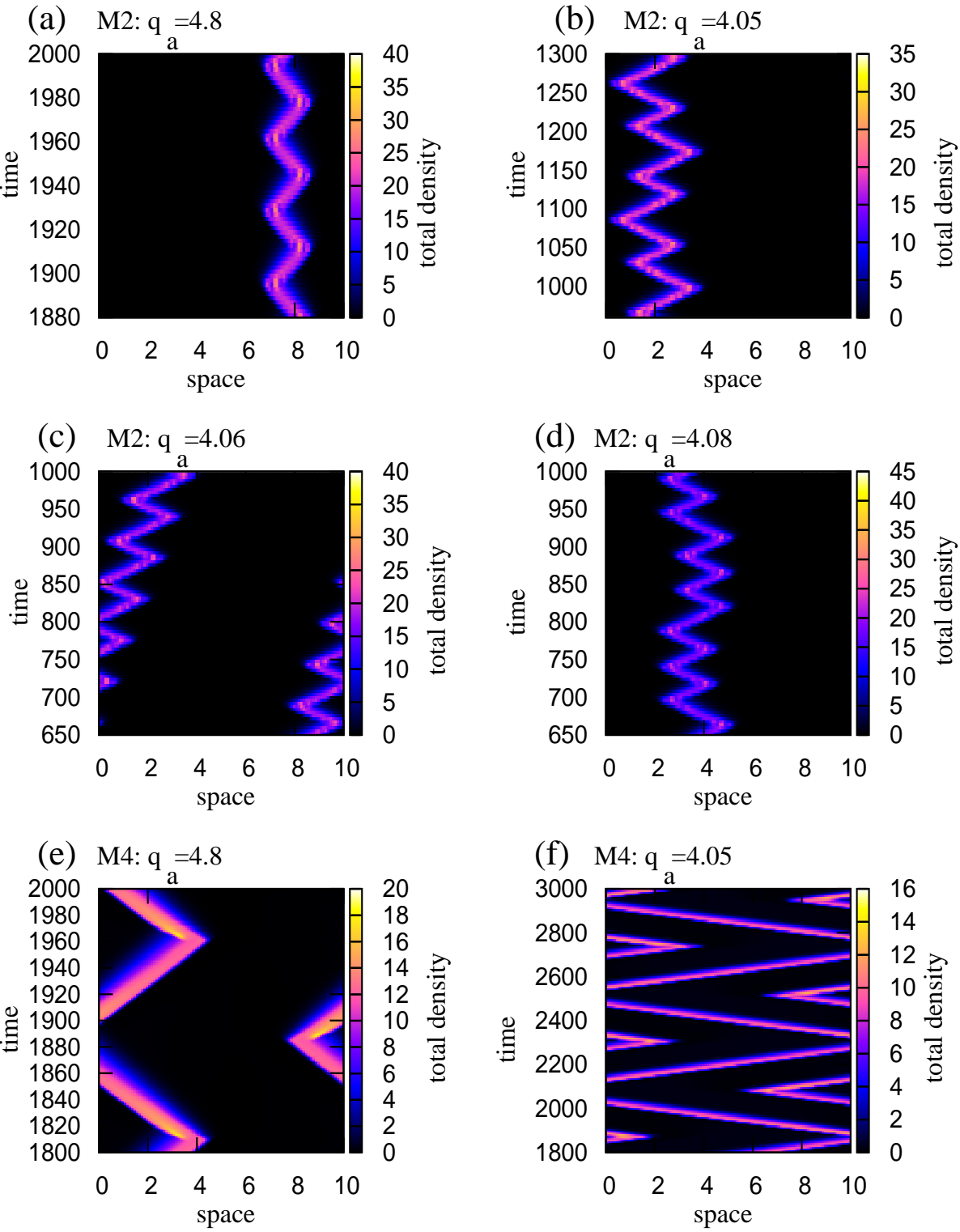

Figure 9. Zigzag pulses exhibited by models M2 and M4. Parameter values are: (a) M2: $q_{a}=4.8$, $q_{r}=1$; (b) M2: $q_{a}=4.05, q_{r}=1$; (c) M2: $q_{a}=4.06, q_{r}=1 ;$ (d) M2: $q_{a}=4.08, q_{r}=1$; (e) M4: $q_{a}=4.8, q_{r}=2$; (f) M4: $q_{a}=4.05, q_{r}=2$. The rest of parameter values are the same for both sub-models: $q_{a l}=2, \gamma=1, \lambda_{1}=0.2, \lambda_{2}=0.9, s_{r}=0.25, s_{a l}=0.5, s_{a}=1.0, L=10$.

Since the zigzags occur only for large $q_{a}$ and the zigzagging groups have very large densities, we can approximate the interaction function by $f \approx 1$ or $f \approx 0$, and thus

$$
\gamma \alpha\left[U, \frac{c_{a}}{\gamma} U\right]-c_{a} \beta\left[U, \frac{c_{a}}{\gamma} U\right] \approx \gamma \lambda_{2}-c_{a}\left(2 \lambda_{1}+\lambda_{2}\right)
$$


(a) model M2

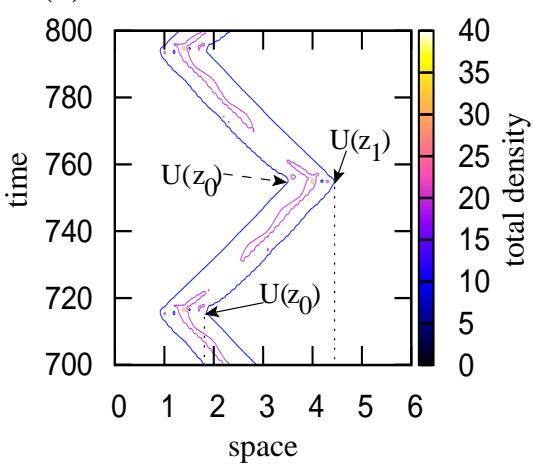

(b) model M4

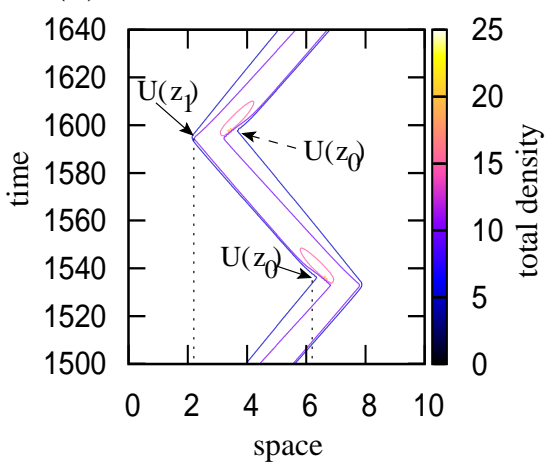

FigURE 10. Surface contour of zigzag pulses exhibited by models (a) M2 and (b) M4. The $z_{0}$ and $z_{1}$ points are some generic space-time points $(z=x-c t)$ at which the groups change their movement direction. Parameter values are: (a) M2: $q_{a}=5.0, q_{r}=1$; (b) M4: $q_{a}=5.0, q_{r}=2$; The rest of parameter values are the same for both sub-models: $q_{a l}=2, \gamma=1, \lambda_{1}=0.2, \lambda_{2}=0.9, s_{r}=0.25$, $s_{a l}=0.5, s_{a}=1.0, L=10$.

Therefore, equation (6.2) becomes

$$
\ln \left(\frac{U\left(z_{1}\right)}{U\left(z_{0}\right)}\right)=\left(z_{1}-z_{0}\right) \frac{\gamma \lambda_{2}-c_{a}\left(2 \lambda_{1}+\lambda_{2}\right)}{\gamma^{2}-c_{a}^{2}}=d \frac{\gamma \lambda_{2}-c_{a}\left(2 \lambda_{1}+\lambda_{2}\right)}{\gamma^{2}-c_{a}^{2}},
$$

where $d=z_{1}-z_{0}$. The reversal time $T$ is thus given by

$$
T=\frac{\ln \left(\frac{U\left(z_{1}\right)}{U\left(z_{0}\right)}\right)\left(\gamma^{2}-c_{a}^{2}\right)}{\gamma \lambda_{2}-c_{a}\left(2 \lambda_{1}+\lambda_{2}\right)} \frac{1}{c_{a}} .
$$

The fact that $T$ is proportional to the logarithm of $U\left(z_{1}\right) / U\left(z_{0}\right)$ is consistent with the argument in [40] (where the authors showed numerically that the reversal time in an individual-based model was proportional to the logarithm of the population density). Since the back of the group slowly disperses as the group moves forward, the densities $U\left(z_{1}\right)$ and $U\left(z_{0}\right)$ (shown in Figure 10) are different, and thus $\ln \left(U\left(z_{1}\right) / U\left(z_{0}\right)\right) \neq 0$. When $c_{a} \rightarrow c$, we obtain $\gamma \lambda_{2}-c_{a}\left(2 \lambda_{1}+\lambda_{2}\right) \rightarrow 0$, and hence $T \rightarrow \infty$ (i.e., there is no turning, and the pattern is described by travelling pulses that move with speed $c$ ).

Figure 11 shows the reversal times for the zigzags exhibited by models M2 and M4, as a function of $q_{a}$. Panels (a) and (b) show the reversal times calculated from the numerical data. When the reversal times are $T \leq 8.86$ (for model M2) and $T \leq 3.8948$ (for model M4), the zigzags are replaced by travelling pulses (with $T \rightarrow \infty$ ). In panels (c) and (d), the red filled circles show the numerical values of $\ln \left(U\left(z_{1}\right) / U\left(z_{0}\right)\right.$ ). The values $U\left(z_{1}\right)$ and $U\left(z_{0}\right)$ are calculated from the data, as in Figure 6 , by recording the maximum value of $U(z)$ within an attraction range of length $z_{a}$ from the front edge of the travelling pulse. (For example, in Figure 6(a) this maximum value is 4.2, while in Figure 6(b) the maximum value is 8.0.) The black filled circles in panels (c) and (d) show the reversal times $T$ obtained by multiplying these logarithms by $\left(\gamma^{2}-c_{a}^{2}\right) /\left(\gamma \lambda_{2}-c_{a}\left(2 \lambda_{1}+\lambda_{2}\right)\right) c_{a}$, for $c_{a}=0.066$. For model M2 (panel (c)), this value of $c_{a}$ seems to be a good approximation of the group speed between the two reversal points. However, for model M4 (panel (d)) this average speed is too low. The open blue circles show the reversal times obtained for $c_{a}=0.0676$. While the reversal times for $q_{a}>5.5$ are similar to the ones in panel (b) (i.e., $T \approx 50$ ), the reversal times for $q_{a}<4.5$ are much lower compared to the ones in panel (b). This difference in the reversal times for small and large $q_{a}$ suggests a dependence of the average speed $c_{a}$ on $q_{a}$. The result makes sense since 
for small $q_{a}$, the groups travel longer before they change direction, and thus the average speed between reversals should be slightly higher in this case.

Finally, we remark in Figure 11 (a),(c) the double values for the reversal times $T$, corresponding to $q_{a} \in[4.05,4.08]$. These double values are the result of non-regular zigzags being displayed by model M2: travelling zigzags $\left(q_{a}=4.06\right)$, or period- $n$ zigzags $\left(n=2\right.$ for $q_{a}=4.06 ; n=4$ for $q_{a}=4.05, q_{a}=4.07$; $n=10$ for $q_{a}=4.08$ ). Model M4 displays travelling zigzags (i.e., period-2 zigzags) only for $q_{a}=4.05$. However, in this case, the two reversal times have relatively close values (i.e., $T=224$ and $T=212$ ) and cannot be distinguished very well on the graphs.
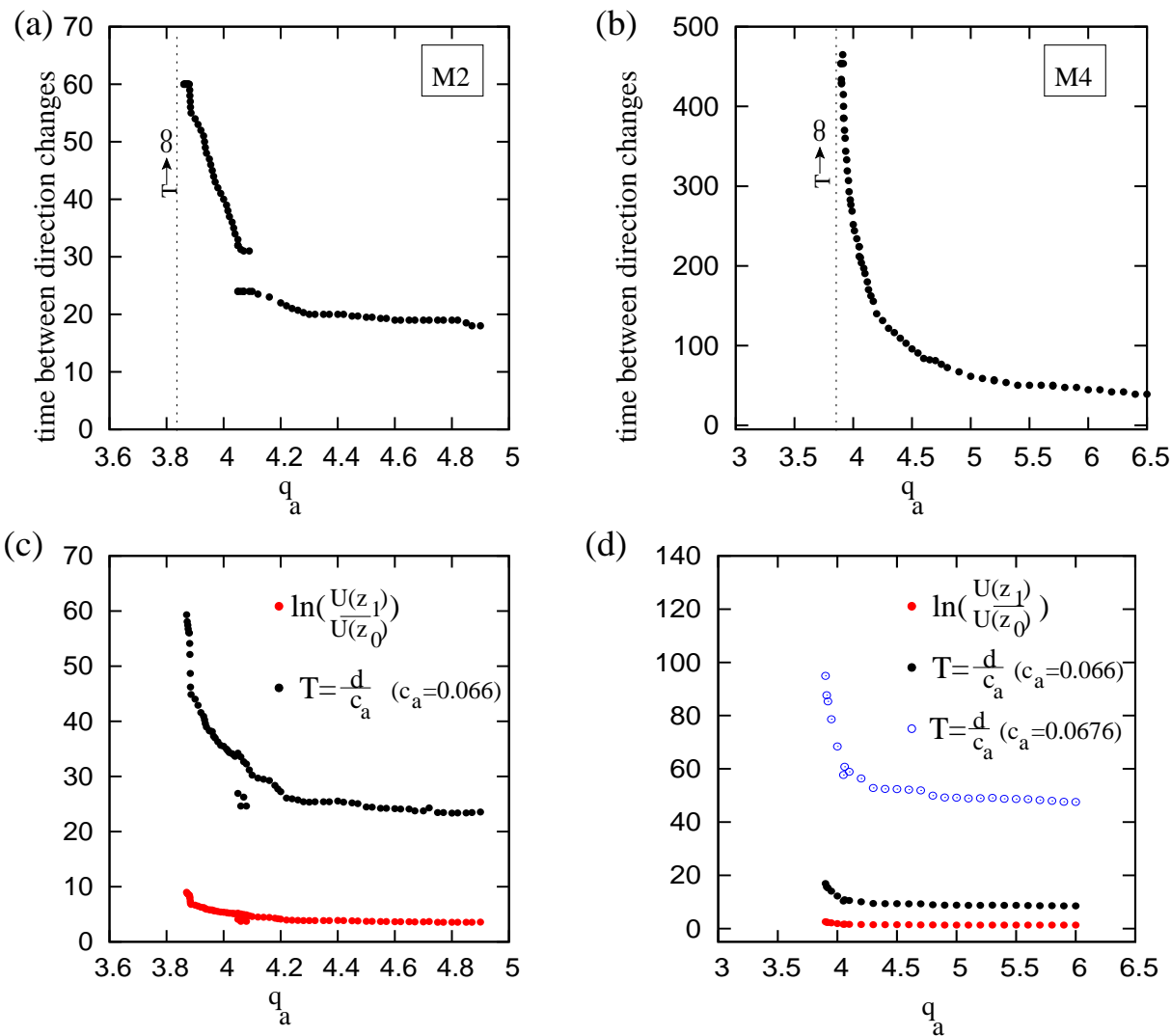

FIGURE 11. Reversal times $T$ for zigzag patterns exhibited by models M2 and M4. (a) Numerically calculated reversal times for model M2; (b) Numerically calculated reversal times for model M4. (c) Model M2: Red (grey - on Black/White prints) filled circles are numerically calculated values for $\ln \left(U\left(z_{1}\right) / U\left(z_{0}\right)\right)$. Black filled circles show the reversal times as given by (6.5), for $c_{a}=0.066$. (d) Model M4: Red filled circles are numerically calculated values for $\ln \left(U\left(z_{1}\right) / U\left(z_{0}\right)\right)$. Black filled circles show the reversal times as given by (6.5), for $c_{a}=0.066$. Blue (dark grey- on Black/White prints) open circles show the reversal times for $c_{a}=0.067$. Parameter values are: (a) M2: $q_{a}=4.05, q_{r}=1$; (b) M2: $q_{a}=5.0, q_{r}=1$; (c) M4: $q_{a}=4.05, q_{r}=2$; (d) M4: $q_{a}=5.0, q_{r}=2$. The rest of parameter values are the same for both sub-models: $q_{a l}=2, \gamma=1, \lambda_{1}=0.2, \lambda_{2}=0.9, s_{r}=0.25, s_{a l}=0.5, s_{a}=1.0$, $L=10$.

\section{Discussion}

In this article, we investigated the role of different communication mechanisms on the structure and speed of moving biological aggregations. To this end, we used a class of nonlocal hyperbolic models 
that allowed for a straightforward incorporation of various communication mechanisms corresponding to different sensory modalities. First, we studied the combined effect of social interactions (attraction, repulsion and alignment) and communication mechanisms on the number of spatially homogeneous steady states. We showed that in the absence of alignment interactions, the formation of polarised steady states as a result of repulsive interactions occurs only when individuals cannot perceive all of their neighbours (e.g., models M4,M5). This suggests that strong repulsion makes individuals align with neighbours by chance. In contrast, the formation of polarised steady states as a result of alignment interactions depends on the magnitude of alignment: for small alignment, those individuals that cannot receive information from all of their neighbours will form polarised states only for some restricted parameter values (see models M3 and M5). In contrast, individuals that receive information from all of their surrounding neighbours will form polarised states for a large range of parameter values. Therefore, the formation of polarised states is influenced by both communication mechanisms and social interactions.

Next, we studied the role of different communication mechanisms on the speed of travelling groups. We approximated the speed values, and showed numerically that groups travel at the maximum speed, independent of the communication mechanism used. (The magnitude of the speed was determined only by the individual turning rates $\lambda_{1}$ and $\lambda_{2}$ and the individual speed $\gamma$ ). This result suggests that for calculating the travelling speed, we could have ignored the nonlocal terms that incorporate communication. However, the presence of these nonlocal terms allowed us to identify the parameter values for which travelling pulses could exist. The inequalities that give the maximum/minimum speed of travelling pulses are only a necessary condition. The existence of a travelling pulse versus a stationary pulse is, in fact, significantly influenced by the magnitude of the attractive interactions (with large attractive interactions leading to stationary pulses in M2). The attractive interactions also seem to affect the transition from a travelling pulse to a zigzagging pulse. For model M4, travelling pulses exist for $q_{a}-q_{r}<2$, while zigzagging pulses exist for $q_{a}-q_{r}>2$. Overall, these results seems to contradict the results in [15], which suggest that a strong attractive interaction can impede the zigzag movement in a 1D individual-based model. However, we note that also for our model, very large attractive interactions (e.g., $q_{a}>10$ ) stop the zigzagging behaviour.

The numerical travelling pulses obtained with models M2 and M4 had different densities and travelled at the same speed. On the other hand, the results presented in Figure 5, which were obtained by assuming the same group density for both M2 and M4 models, showed that the M2 and M4 pulses could travel at different speeds. To check whether the M2 and M4 groups that start with the same initial densities move at different speeds, we performed numerical simulations with rectangle-shaped initial conditions that had the same maximum density ( $B=4$; not shown here). The results showed that just before groups started travelling, the densities of the groups changed (by dispersing or contracting). As groups started moving, their speed increased quickly towards the maximum speed $c \approx 0.0692$. This result raises the question of whether there is a connection between the communication mechanism used and the group density necessary to ensure movement at maximum speed.

While communication mechanisms did not influence the magnitude of the travelling speed, they seemed to have an influence on the zigzagging behaviour. In particular, the reversals in the movement direction were less frequent when individuals did not receive information from all of their neighbours (model M4). In contrast, when individuals received information from all of their neighbours (model M2), the groups were formed of high densities of individuals, and the reversals occurred more frequently. The results presented here raise the question of whether the transition from short moves to long moves (observed, for example, in foraging aggregations) could also be the result of defects in the inter-individual communication (due to external factors, such as wind - which distorts sounds signals, or vegetation - which impacts visual communication).

Overall, our findings add to the existent theoretical results on the importance of group-level communication to the structure and movement of biological aggregations [17]. Although the role of individual-level communication on individual movement can be investigated experimentally [39], we are still far from understanding experimentally the role of group-level communication on the movement of animal aggre- 
gations. Thus, the mathematical modelling of group-level animal communication and the subsequent analysis of these models are among the few tools that we currently have to formulate hypotheses about the possible biological mechanisms behind the observed group behaviours. Further research should be directed to develop new experimental methods to investigate how group-level communication influences the movement of the group. From a mathematical perspective, it is necessary to develop new models that incorporate more detail about the communication signals (e.g., by differentiating signals used for directional group coordination from signals used for group cohesion).

\section{References}

[1] M. Aldana, C. Huepe, Phase transitions in self-driven many-particle systems and related non-equilibrium models: a network approach, J. Stat. Phys., 112 (2003), No. 1-2, 135-153.

[2] I. Aoki, A simulation study on the schooling mechanism in fish, Bull. Japan Soc. Sci. Fish, 48 (1982), 1081-1088.

[3] S. Bazazi, F. Bartumeus, J.J. Hale, I.D. Couzin, Intermittent motion in desert locusts: behavioural complexity in simple environments, PLOS Comput. Biol., 8 (2012), No. 5, e1002498.

[4] N.W.F. Bode, D.W. Franks, A.J. Wood, Making noise: emergent stochasticity in collective motion, J. Theor. Biol., 267 (2010), No. 3, 292-299.

[5] N.W.F. Bode, A.J. Wood, D.W. Franks, The impact of social networks on animal collective motion, Anim. Behav., 82 (2011), 29-38.

[6] C.A.H. Bousquet, D.J.T. Sumpter, M.B. Manser, Moving calls: a vocal mechanism underlying quorum decisions in cohesive groups, Proc. R. Soc. B, 278 (2011), No. 1711, 1482-1488.

[7] C. Brown, K.N. Laland, Social learning in fishes: a review, Fish and fisheries, 4 (2003), 280-288.

[8] J. Buhl, D. J. T. Sumpter, I. D. Couzin, J. J. Hale, E. Despland, E. R. Miller, S. J. Simpson, From disorder to order in marching locusts, Science, 312 (2006), 1402-1406.

[9] J. Buhl, G.A. Sword, S. J. Simpson, Using field data to test locust migratory band collective movement models, Interface Focus, 2 (2012), No. 6, 757-763.

[10] P.-L. Buono, R. Eftimie, Analysis of Hopf-Hopf bifurcations in nonlocal hyperbolic models for self-organised aggregations, Math. Models Methods Appl. Sci. (2013), To Appear.

[11] G. Chaverri, E.H. Gillam, T.H. Kunz, A call-and-response system facilitates group cohesion among disc-winged bats, Behav. Ecol., 24 (2013), No. 2, 481-487.

[12] I. D. Couzin, J. Krause, R. James, G.D. Ruxton, N. R. Franks, Collective memory and spatial sorting in animal groups, J. Theor. Biol., 218 (2002), 1-11.

[13] A. Czirók, A.-L. Barabási, T. Vicsek, Collective motion of self-propelled particles: kinetic phase transition in one dimension, Physical Review Letters, 82 (1999), No. 1, 209-212.

[14] M.R. D'Orsogna, Y.L. Chuang, A.L. Bertozzi, L.S. Chayes, Self-propelled particles with soft-core interactions: patterns, stability and collapse, Phys. Rev. Lett., 96 (2006), No. 10, 104302.

[15] V. Dossetti, Cohesive motion in one-dimensional flocking, J. Phys. A: Math. Theor., 45 (2012), 035003.

[16] R. Eftimie, Hyperbolic and kinetic models for self-organized biological aggregations and movement: a brief review, J. Math. Biol., 65 (2012), No. 1, 35-75.

[17] R. Eftimie, G. de Vries, M. A. Lewis, Complex spatial group patterns result from different animal communication mechanisms, Proc. Natl. Acad. Sci., 104 (2007), No. 17, 6974-6979.

[18] R. Eftimie, G. de Vries, M. A. Lewis, F. Lutscher, Modeling group formation and activity patterns in self-organizing collectives of individuals, Bull. Math. Biol., 69 (2007), No. 5, 1537-1566.

[19] R. Eftimie, G. de Vries, M.A. Lewis, Weakly nonlinear analysis of a hyperbolic model for animal group formation, J. Math. Biol., 59 (2009), 37-74.

[20] R. Erban, H. G. Othmer, From individual to collective behavior in bacterial chemotaxis, SIAM J. Appl. Math., 65 (2004), No. 2, 361-391.

[21] R. Fetecau, R. Eftimie, An investigation of a nonlocal hyperbolic model for self-organization of biological groups, J. Math. Biol., 61 (2009), No. 4, 545-579.

[22] R.C. Fetecau, Collective behavior of biological aggregations in two dimensions: a nonlocal kinetic model, Math. Model. Method. Appl. Sci., 21 (2011), No. 07, 1539.

[23] V. Gazi, K.M. Passino, A class of attraction/repulsion functions for stable swarm aggregations, Int. J. Control, 77 (2004), No. 18, 1567-1579.

[24] S. Gueron, S. A. Levin, D. I. Rubenstein, The dynamics of herds: from individuals to aggregations, J. Theor. Biol., $182(1996), 85-98$.

[25] C. K. Hemelrijk, H. Kunz, Density distribution and size sorting in fish schools: an individual-based model, Behav. Ecol., 16 (2005), No. 1, 178-187.

[26] T. Hillen, Invariance principles for hyperbolic random walk systems, J. Math. Ana. Appl., 210 (1997), 360-374.

[27] D.J. Hoare, I.D. Couzin, J.G. Godin, J. Krause, Context-dependent group size choice in fish, Anim. Behav., 67 (2004), $155-164$.

[28] D. Horstmann, A. Stevens, A constructive approach to traveling waves in chemotaxis, J. Nonlinear. Sci., 14 (2004), No. 1, 1-25. 
[29] A. Huth, C. Wissel, The simulation of the movement of fish schools, J. Theor. Biol., 156 (1992), $365-385$.

[30] _ The simulation of fish schools in comparison with experimental data, Ecol. Model., 75/76 (1994), 135-145.

[31] Y. Inada, Steering mechanisms of fish schools, Complexity International, 8 (2001), 1-9.

[32] M. Iwasa, K. Iida, D. Tanaka, Hierarchical cluster structures in a one-dimensional swarm oscillator model, Phys. Rev. E, 81 (2010), No. 4, 046220.

[33] E.F. Keller, L.A. Segel, Traveling bands of chemotactic bacteria: A theoretical analysis, J. Theor. Biol., 30 (1971), 235-248.

[34] G. Kerth, C. Ebert, C. Schmidtke, Group decision making in fission-fusion societies: evidence from two-field experiments in Bechstein's bats, Proc. R. Soc. B, 273 (2006), 2785-2790.

[35] H. Levine, W.-J. Rappel, I. Cohen, Self-organization in systems of self-propelled particles, Phys. Rev. E, 63 (2000), No. 1, 01701.

[36] R. Lui, Z.A. Wang, Travelling wave solutions from microscopic to macroscopic chemotaxis models, J. Math. Biol., 61 (2010), No. 5, 739-761.

[37] R. Lukeman, Y.-X. Li, L. Edelstein-Keshet, Inferring individual rules from collective behaviour, Proc. Natl. Acad. Sci., 107 (2010), No. 28, 12576-12580.

[38] T. Nagai, T. Ikeda, Travelling waves in a chemotactic model, J. Math. Biol., 30 (1991), No. 2, $169-184$.

[39] S.T.D. New, R.A. Peters, A framework for quantifying properties of 3-dimensional movement-based signals, Current Zoology, 56 (2010), No. 3, 327-336.

[40] O.J. O'Loan, M.R. Evans, Alternating steady state in one-dimensional flocking, J. Phys A: Math. Gen., 32 (1999), No. 8, L99.

[41] J. K. Parrish, S. V. Viscido, D. Grunbaum, Self-organized fish schools: An examination of emergent properties, Bioll. Bull., 202 (2002), 296-305.

[42] B. Pfistner, A one dimensional model for the swarming behaviour of Myxobacteria, Biological Motion, Lecture Notes on Biomathematics, 89 (W. Alt, G. Hoffmann, eds.), Springer, 1990, pp. 556-563.

[43] H. Pomeroy, F. Heppner, Structure of turning in airborne rock dove (Columba Livia) flocks, The Auk, 109 (1992), 256-267.

[44] J.R. Raymond, M.R. Evans, Flocking regimes in a simple lattice model, Phys. Rev. E Stat. Nonlin. Soft Matter Phys., 73 (2006), No. 3/2, 036112.

[45] H. Reuter, B. Breckling, Self organization of fish schools: an object-oriented model, Ecol. Model., 75/76 (1994), 147159.

[46] C. W. Reynolds, Flocks, herds and schools: A distributed behavioral model, Computer Graphics, 21 (1987), $25-34$.

[47] J. Saragosti, V. Calvez, N. Bournaveas, A. Buguin, P. Silberzanand B. Perthame, Mathematical description of bacterial traveling pulses, PLOS Computational Biology, 6 (2010), No. 8, e1000890.

[48] H.R. Schwetlick, Travelling waves for chemotaxis-systems, Proc. Appl. Math. Mech, 3 (2003), 476-478.

[49] S. Stöcker, Models for tuna school formation, Math. Biosci, 156 (1999), 167-190.

[50] C. M. Topaz, A. L. Bertozzi, Swarming patterns in a two-dimensional kinematic model for biological groups, SIAM J. Appl. Math., 65 (2006), No. 1, 152-174.

[51] C. M. Topaz, A. L. Bertozzi, M. A. Lewis, A nonlocal continuum model for biological aggregation, Bull. Math. Bio., 68 (2006), 1601-1623.

[52] C. Torney, Z. Neufeld, I. D. Couzin, Context-dependent interaction leads to emergent search behaviour in social aggregates, Proc. Natl. Acad. Sci., 106 (2009), No. 52, 22055-22060.

[53] S. V. Viscido, J. K. Parish, D. Grunbaum, Individual behavior and emergent properties of fish schools: a comparison of observation and theory, Mar. Ecol. Prog. Ser., 273 (2004), 239-249.

[54] C. Xue, H.J. Hwang, K.J. Painter, R. Erban, Travelling waves in hyperbolic chemotactic equations, Bull. Math. Biol., 73 (2011), No. 8, 1695-1733. 